\title{
Haar Wavelet Method for the System of Integral Equations
}

\author{
Hassan A. Zedan ${ }^{1,2}$ and Eman Alaidarous ${ }^{1}$ \\ ${ }^{1}$ Mathematics Department, Faculty of Science, King Abdulaziz University, P.O. Box 80203, Jeddah 21589, Saudi Arabia \\ ${ }^{2}$ Mathematics Department, Faculty of Science, Kafrelsheikh University, Kafrelsheikh, Egypt
}

Correspondence should be addressed to Hassan A. Zedan; hassanzedan2003@yahoo.com

Received 15 March 2014; Revised 13 May 2014; Accepted 20 May 2014; Published 21 July 2014

Academic Editor: Imran Naeem

Copyright (C) 2014 H. A. Zedan and E. Alaidarous. This is an open access article distributed under the Creative Commons Attribution License, which permits unrestricted use, distribution, and reproduction in any medium, provided the original work is properly cited.

We employed the Haar wavelet method to find numerical solution of the system of Fredholm integral equations (SFIEs) and the system of Volterra integral equations (SVIEs). Five test problems, for which the exact solution is known, are considered. Comparison of the results is obtained by the Haar wavelet method with the exact solution.

\section{Introduction}

The Haar wavelet was initiated and independently developed by Haar [1] in the early nineteen tens. In recent years, many different methods and different basis functions have been used to estimate the solution of the system of integral equations, such as Adomian decomposition method [1, 2], Taylor's expansion method [3, 4], homotopy perturbation method [5, 6], projection method and Nystrom method [7], Spline collocation method [8], Runge-Kutta method [9], sinc method [10], Tau method [11], block-pulse functions, hat basis functions method [12], and operational matrices [13, 14].

In the present paper, we use Haar wavelet method to solve the system of linear Fredholm integral equations (SLFIEs) of the second kind:

$$
\mathbf{U}(x)-\int_{0}^{1} \mathbf{K}(x, t) \mathbf{U}(t) d t=\mathbf{F}(x), \quad 0<x<1,
$$

and the system of linear Volterra integral equations (SLVIEs) of the second kind:

$$
\mathbf{U}(x)-\int_{0}^{x} \mathbf{K}(x, t) \mathbf{U}(t) d t=\mathbf{F}(x), \quad 0<x<1,
$$

where

$$
\begin{aligned}
& \mathbf{U}(x)=\left[u_{1}(x), u_{2}(x), \ldots, u_{n}(x)\right]^{T}, \\
& \mathbf{F}(x)=\left[f_{1}(x), f_{2}(x), \ldots, f_{n}(x)\right]^{T}, \\
& \mathbf{K}(x, t)=k_{i j}(x, t), \quad i, j=1,2 \ldots, n .
\end{aligned}
$$

In (1) and (2), the functions $\mathbf{K}$ and $\mathbf{F}$ are given, and $\mathbf{U}$ is the vector function of the solution of systems (1) and (2) that will be determined. Also, we assume that (1) and (2) have a unique solution.

\section{Haar Wavelet Method}

Let us confine to the time interval $t \in[0,1]$. The Haar wavelet family is

$$
h_{i}(x)= \begin{cases}1 & \text { for } t \in\left[\tau_{1}, \tau_{2}\right] \\ -1 & \text { for } t \in\left[\tau_{2}, \tau_{3}\right] \\ 0 & \text { elsewhere. }\end{cases}
$$

Here the notations are as follows:

$$
\tau_{1}=\frac{k}{m}, \quad \tau_{2}=\frac{k+(1 / 2)}{m}, \quad \tau_{3}=\frac{k+1}{m} .
$$


The integer $m=2^{j}, j=0,1, \ldots, J$, indicates the level of the wavelet; $k=0,1, \ldots, m-1$ is the translation parameter. The integer $J$ determines the maximal level of resolution. The index $i$ is calculated from the formula $i=m+k+1$; the minimal value for which (4) holds is $i=2$ (then $m=1, k=$ 0 ); the maximal value is $i=2 M$, where $M=2^{J}$. The index $i=1$ corresponds to the scaling function of the Haar wavelet $h_{1}(t) \equiv 1$.

Simple calculations show the following.

We have that

$$
\int_{0}^{1} h_{i}(t) h_{l}(t) d t= \begin{cases}\frac{1}{m} & \text { for } i=l \\ 0 & \text { for } i \neq l\end{cases}
$$

consequently, the functions $h_{i}(t)$ are orthogonal.

Next we discretize the functions $h_{i}(x)$ by dividing the interval $x \in[0,1]$ into $2 M$ parts of equal length $\Delta x=1 /(2 M)$ and introduce the collocation points:

$$
x_{l}=\frac{l-(1 / 2)}{2 M}, \quad l=1,2, \ldots, 2 M .
$$

Following Chen and Hsiao $[15,16]$ the coefficients matrix $H_{i l}=h_{i}\left(t_{l}\right)$ is introduced (this is a $2 M \times 2 M$ matrix). A function $u(t)$ which is defined in the interval $x \in[0,1]$ can be expanded into the Haar wavelet series:

$$
u(x)=\sum_{i=1}^{2 M} a_{i} h_{i}(x),
$$

where $a$ is the wavelet coefficients. The discrete form of this equation is

$$
u\left(x_{l}\right)=\sum_{i=1}^{2 M} a_{i} h_{i}\left(x_{l}\right)=\sum_{i=1}^{2 M} a_{i} H_{i l}
$$

or in a matrix presentation $u=a H$, where $u$ and $a$ are $2 M$ dimensional row vectors.

\section{System of Fredholm Integral Equation}

Let us consider SLFIEs ( 1$)$ at $(i, j, n=1,2)$ :

$$
\begin{array}{r}
u(x)-\int_{0}^{1} k_{11}(x, t) u(t) d t-\int_{0}^{1} k_{12}(x, t) v(t) d t=f_{1}(x), \\
v(x)-\int_{0}^{1} k_{21}(x, t) u(t) d t-\int_{0}^{1} k_{22}(x, t) v(t) d t=f_{2}(x), \\
x \in[0,1] .
\end{array}
$$

Let

$$
u(x)=\sum_{i=1}^{2 M} a_{i} h_{i}(x), \quad v(x)=\sum_{i=1}^{2 M} b_{i} h_{i}(x) .
$$

Replacing (12) into (10) and (11) we find

$$
\begin{aligned}
& \sum_{i=1}^{2 M} a_{i} h_{i}(x)-\sum_{i=1}^{2 M} a_{i} G_{1 i}(x)-\sum_{i=1}^{2 M} b_{i} G_{2 i}(x)=f_{1}(x), \\
& \sum_{i=1}^{2 M} b_{i} h_{i}(x)-\sum_{i=1}^{2 M} a_{i} G_{3 i}(x)-\sum_{i=1}^{2 M} b_{i} G_{4 i}(x)=f_{2}(x),
\end{aligned}
$$

where

$$
\begin{aligned}
& G_{1 i}(x)=\int_{0}^{1} k_{11}(x, t) h_{i}(t) d t, \\
& G_{2 i}(x)=\int_{0}^{1} k_{12}(x, t) h_{i}(t) d t, \\
& G_{3 i}(x)=\int_{0}^{1} k_{21}(x, t) h_{i}(t) d t, \\
& G_{4 i}(x)=\int_{0}^{1} k_{22}(x, t) h_{i}(t) d t .
\end{aligned}
$$

Next we will evaluate the wavelet coefficients $a_{i}$ and $b_{i}$ in the following way.

Collocation Method. Satisfying (12) only at the collocation points (7) we get a system of linear equations:

$$
\begin{array}{r}
\sum_{i=1}^{2 M}\left[a_{i}\left(h_{i}\left(t_{l}\right)-G_{1 i}\left(x_{l}\right)\right)-b_{i} G_{2 i}\left(x_{l}\right)\right]=f_{1}\left(x_{l}\right), \\
\sum_{i=1}^{2 M}\left[b_{i}\left(h_{i}\left(t_{l}\right)-G_{4 i}\left(x_{l}\right)\right)-a_{i} G_{3 i}\left(x_{l}\right)\right]=f_{2}\left(x_{l}\right), \\
l=1,2, \ldots, 2 M .
\end{array}
$$

Therefore the matrix form of this system is given by

$a\left(H-G_{1}\right)-b G_{2}=F_{1}, \quad b\left(H-G_{4}\right)-a G_{3}=F_{2}$,

where $\mathbf{G}_{i l}=\mathbf{G}_{i}\left(x_{l}\right), \mathbf{F}_{l}=\mathbf{f}\left(x_{l}\right)$.

Now, we can present the following problems.

Example 1. Consider the following SLFIEs $[3,16]$ :

$$
\begin{gathered}
u(x)-\int_{0}^{1}(x-t)^{3} u(t) d t-\int_{0}^{1}(x-t)^{2} v(t) d t \\
=\frac{1}{20}-\frac{11}{30} x+\frac{5}{3} x^{2}-\frac{1}{3} x^{3}, \\
v(x)-\int_{0}^{1}(x-t)^{4} u(t) d t-\int_{0}^{1}(x-t)^{3} v(t) d t \\
=-\frac{1}{30}-\frac{41}{60} x+\frac{3}{20} x^{2}+\frac{23}{12} x^{3}-\frac{1}{3} x^{4} .
\end{gathered}
$$


Carrying out the integration in (13) we obtain

$$
\begin{aligned}
& G_{1}(x) \\
& \quad= \begin{cases}x\left(1+x^{2}\right)-\frac{1+6 x^{2}}{4} & \text { for } i=1 \\
\frac{7-24 m x+24\left(k+k^{2}-2 k m x+m^{2} x^{2}\right)}{32 m^{4}} & \text { for } i>1,\end{cases}
\end{aligned}
$$

$G_{2}(x)$

$$
= \begin{cases}\frac{1}{3}+(x-1) x & \text { for } i=1 \\ -\frac{1+2 k-2 m x}{4 m^{3}} & \text { for } i>1,\end{cases}
$$

$$
\begin{array}{r}
G_{3}(x) \\
=\left\{\begin{array}{cc}
\frac{1}{5}+x(x-1)(1+x(x-1)) & \text { for } i=1 \\
-\left(( 1 + 2 k - 2 m x ) \left(3+8 k^{2}+k(8-16 m x)\right.\right. \\
\times\left(16 m^{5}\right)^{-1} & +8 m x(-1+m x)))
\end{array}\right. \\
\end{array}
$$

$$
\begin{aligned}
& G_{4}(x) \\
& \quad= \begin{cases}x\left(1+x^{2}\right)-\frac{1+6 x^{2}}{4} & \text { for } i=1 \\
\frac{7-24 m x+24\left(k+k^{2}-2 k m x+m^{2} x^{2}\right)}{32 m^{4}} & \text { for } i>1 .\end{cases}
\end{aligned}
$$

We apply collocation method, and vectors $a_{i}$ and $b_{i}$ can be calculated from (15); the functions $u(x)$ and $v(x)$ are evaluated from (11).

Computations were carried out for different values of $J$. These results were compared with the exact solution $u(x)=$ $x^{2}$ and $v(x)=-x+x^{2}+x^{3}$.

The accuracy of the results (see Table 1 and Figure 1) was estimated by the error function for $u(x)$ and $v(x)$ :

$$
\begin{aligned}
& e_{J}=\max _{1 \leq l \leq 2 M}\left|u\left(x_{l}\right)-u_{e x}\left(x_{l}\right)\right|, \\
& e_{J}=\max _{1 \leq l \leq 2 M}\left|v\left(x_{l}\right)-v_{e x}\left(x_{l}\right)\right|,
\end{aligned}
$$

where $x_{l}$ is defined by (7).

Example 2. Consider the following SLFIEs given by $[10,12]$ :

$$
\begin{aligned}
& u(x)+\int_{0}^{1} e^{x-t} u(x) d t+\int_{0}^{1} e^{(x+2) t} v(x) d t \\
& =2 e^{x}+\frac{e^{x+1}-1}{x+1} \\
& v(x)+\int_{0}^{1} e^{x t} u(x) d t+\int_{0}^{1} e^{x+t} v(x) d t \\
& =e^{x}+e^{-x}+\frac{e^{x+1}-1}{x+1} .
\end{aligned}
$$

TABLE 1: Error of $u(x)$ and $v(x)$ of Example 1.

\begin{tabular}{cccc}
\hline$J$ & $2 M$ & Error of function $u(x)$ & Error of function $v(x)$ \\
\hline 2 & 8 & $1.45 E-3$ & $1.57 E-3$ \\
3 & 16 & $3.65 E-4$ & $4.16 E-4$ \\
4 & 32 & $9.19 E-5$ & $1.06 E-4$ \\
5 & 64 & $2.29 E-5$ & $2.68 E-5$ \\
\hline
\end{tabular}

Carrying out the integration in (13) we obtain

$$
\begin{aligned}
& G_{1}(x)= \begin{cases}(-1+e) e^{-1+x} & \text { for } i=1 \\
e^{-((1+k) / m)+x}\left(-1+e^{1 / 2 m}\right)^{2} & \text { for } i>1,\end{cases} \\
& G_{2}(x)= \begin{cases}\frac{-1+e^{2+x}}{2+x} & \text { for } i=1 \\
-\frac{e^{k(2+x) / m}\left(-1+e^{(2+x) / 2 m}\right)^{2}}{2+x} & \text { for } i>1,\end{cases} \\
& G_{3}(x)= \begin{cases}\frac{-1+e^{x}}{x} & \text { for } i=1 \\
-\frac{x-e^{k x / m}\left(-1+e^{x / 2 m}\right)^{2} / x}{4 m^{2}} & \text { for } i>1,\end{cases} \\
& G_{4}(x)= \begin{cases}(-1+e) e^{x} & \text { for } i=1 \\
-e^{(k / m)+x}\left(-1+e^{1 / 2 m}\right)^{2} & \text { for } i>1 .\end{cases}
\end{aligned}
$$

We apply collocation method, and vectors $a_{i}$ and $b_{i}$ can be calculated from (15); the functions $u(x)$ and $v(x)$ are evaluated from (11).

Computations were carried out for different values of $J$. These results were compared with the exact solution (see Table 2 and Figure 2) $u(x)=e^{x}$ and $v(x)=e^{-x}$.

Example 3. Consider the following SLFIEs given by [10]:

$$
\begin{aligned}
& u(x)-\int_{0}^{1} \frac{x+t}{3} u(t) d t-\int_{0}^{1} \frac{x+t}{3} v(t) d t=\frac{x}{18}+\frac{17}{36}, \\
& v(x)-\int_{0}^{1} x t u(t) d t-\int_{0}^{1} x t v(t) d t=x^{2}-\frac{19}{12}+1 .
\end{aligned}
$$

Carrying out the integration in (13) we obtain

$$
G_{j}(x)=\left\{\begin{array}{l}
\frac{1}{6}+\frac{x}{3} \text { for } i=1 \\
-\frac{1}{12 m^{2}} \text { for } i>1
\end{array} j=1,2,3,4\right.
$$

We apply collocation method, and vectors $a_{i}$ and $b_{i}$ can be calculated from (15); the functions $u(x)$ and $v(x)$ are evaluated from (11).

Computations were carried out for different values of $J$ (see Table 3 and Figure 3). These results were compared with the exact solution $u(x)=x+1$ and $v(x)=x^{2}+1$.

By comparing the results obtained with the results found in $[3,10,12]$, we find that the results we have obtained are accurate and error rate, which is much less but almost 


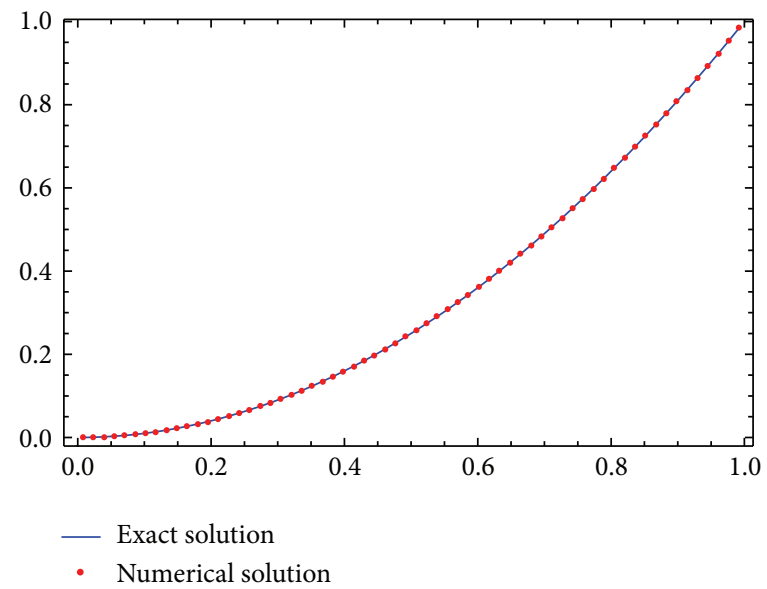

(a)

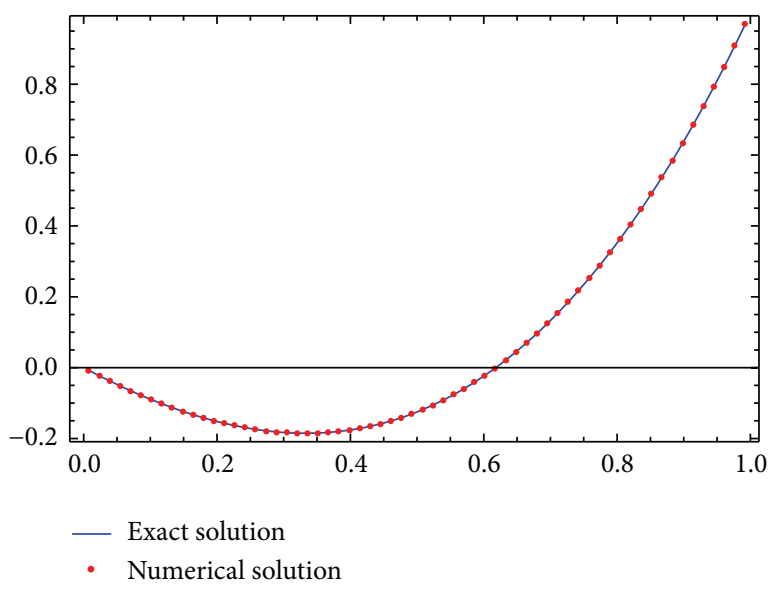

(b)

Figure 1: (a) Exact and Haar wavelet solution of $u(x)$ at $J=5$. (b) Exact and Haar wavelet solution of $v(x)$ at $J=5$.

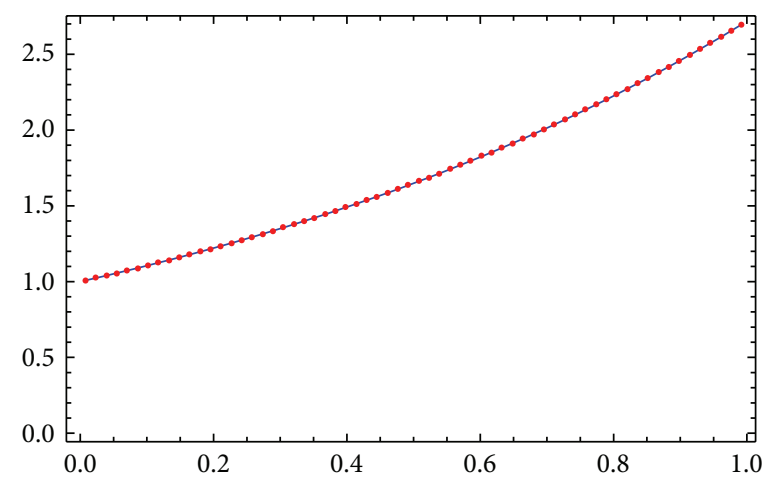

_ Exact solution

- Numerical solution

(a)

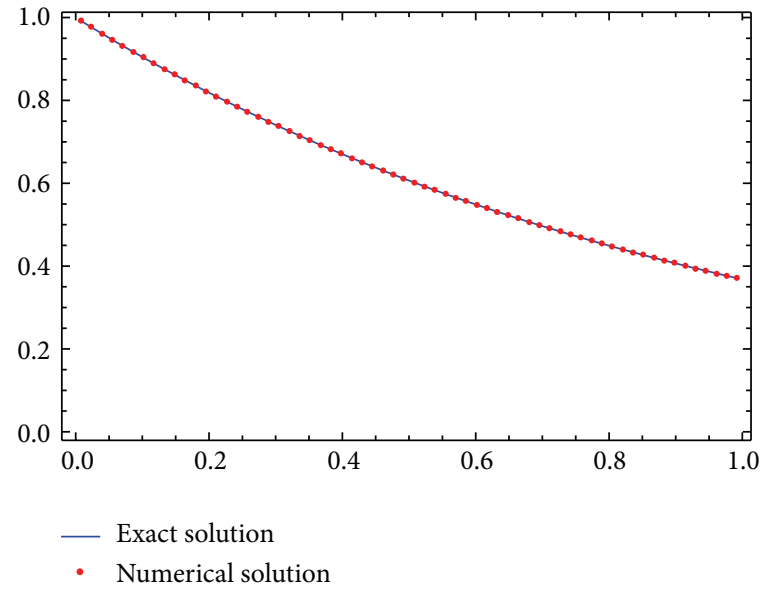

(b)

Figure 2: (a) Exact and Haar wavelet solution of $u(x)$ at $J=5$. (b) Exact and Haar wavelet solution of $v(x)$ at $J=5$.

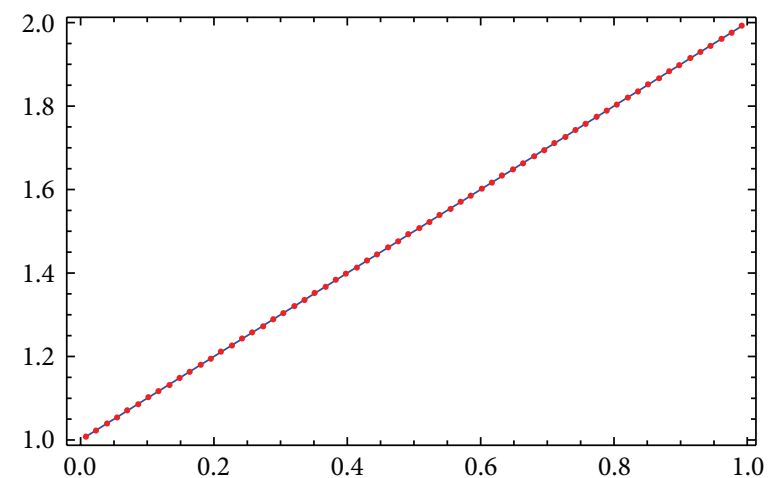

- Exact solution

- Numerical solution

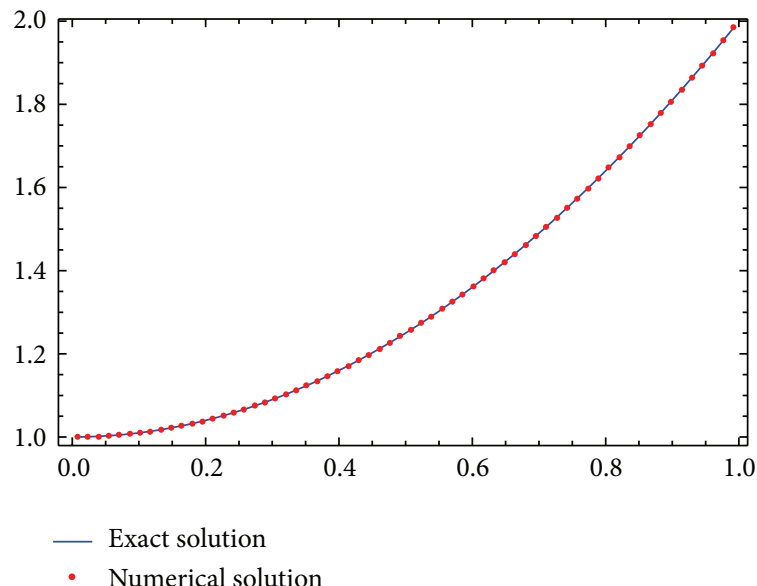

(b)

Figure 3: (a) Exact and Haar wavelet solution of $u(x)$ at $J=5$. (b) Exact and Haar wavelet solution of $v(x)$ at $J=5$. 
TABLE 2: Error of $u(x)$ and $v(x)$ of Example 2.

\begin{tabular}{cccc}
\hline$J$ & $2 M$ & Error of function $u(x)$ & Error of function $v(x)$ \\
\hline 2 & 8 & $2.28 E-2$ & $7.30 E-3$ \\
3 & 16 & $5.79 E-3$ & $1.82 E-3$ \\
4 & 32 & $1.45 E-3$ & $4.56 E-4$ \\
5 & 64 & $2.95 E-4$ & $4.62 E-5$ \\
\hline
\end{tabular}

TABLE 3: Error of $u(x)$ and $v(x)$ of Example 3.

\begin{tabular}{cccc}
\hline$J$ & $2 M$ & Error of function $u(x)$ & Error of function $v(x)$ \\
\hline 2 & 8 & $6.15 E-3$ & $8.22 E-3$ \\
3 & 16 & $1.57 E-3$ & $2.13 E-3$ \\
4 & 32 & $3.96 E-4$ & $5.42 E-4$ \\
5 & 64 & $9.95 E-5$ & $1.36 E-4$ \\
\hline
\end{tabular}

nonexistent compared to other methods, is also shown in tables of error attached and this shows the importance of the method used.

\section{System of Volterra Integral Equations}

Let us consider system of linear Volterra integral equations (SLVIEs) (2) at $(i, j=1,2, n=2)$ :

$$
\begin{array}{r}
u(x)-\int_{0}^{x} k_{11}(x, t) u(t) d t-\int_{0}^{x} k_{12}(x, t) v(t) d t=f_{1}(x) \\
v(x)-\int_{0}^{x} k_{21}(x, t) u(t) d t-\int_{0}^{x} k_{22}(x, t) v(t) d t=f_{2}(x) \\
x \in[0,1]
\end{array}
$$

Its discrete form is

$$
\begin{array}{r}
u\left(x_{l}\right)-\int_{0}^{x_{l}} k_{11}\left(x_{l}, t\right) u(t) d t \\
-\int_{0}^{x_{l}} k_{12}\left(x_{l}, t\right) v(t) d t=f_{1}\left(x_{l}\right), \\
v\left(x_{l}\right)-\int_{0}^{x_{l}} k_{21}\left(x_{l}, t\right) u(t) d t \\
-\int_{0}^{x_{l}} k_{22}\left(x_{l}, t\right) v(t) d t=f_{2}\left(x_{l}\right), \\
x \in[0,1],
\end{array}
$$

where $x_{l}$ defined in (7) are the collocation points.

Let

$$
u(x)=\sum_{i=1}^{2 M} a_{i} h_{i}(x), \quad v(x)=\sum_{i=1}^{2 M} b_{i} h_{i}(x)
$$

Replacing (25) with (24) we find

$$
\begin{aligned}
& \sum_{i=1}^{2 M} a_{i} h_{i}\left(x_{l}\right)-\sum_{i=1}^{2 M} a_{i}\left(G_{11}\right)_{i}\left(x_{l}\right)-\sum_{i=1}^{2 M} b_{i}\left(G_{12}\right)_{i}\left(x_{l}\right)=f_{1}\left(x_{l}\right), \\
& \sum_{i=1}^{2 M} b_{i} h_{i}\left(x_{l}\right)-\sum_{i=1}^{2 M} a_{i}\left(G_{21}\right)_{i}\left(x_{l}\right)-\sum_{i=1}^{2 M} b_{i}\left(G_{22}\right)_{i}\left(x_{l}\right)=f_{2}\left(x_{l}\right) .
\end{aligned}
$$

The matrices $\left(G_{s r}\right)_{i l}=\left(G_{s}\right)_{i}\left(x_{l}\right), s, r=1,2$, are now defined as

$$
\begin{aligned}
& \left(G_{11}\right)_{i l}=\int_{0}^{x_{l}} k_{11}\left(x_{l}, t\right) h_{i}(t) d t, \\
& \left(G_{12}\right)_{i l}=\int_{0}^{x_{l}} k_{12}\left(x_{l}, t\right) h_{i}(t) d t, \\
& \left(G_{21}\right)_{i l}=\int_{0}^{x_{l}} k_{21}\left(x_{l}, t\right) h_{i}(t) d t, \\
& \left(G_{22}\right)_{i l}=\int_{0}^{x_{l}} k_{22}\left(x_{l}, t\right) h_{i}(t) d t .
\end{aligned}
$$

By computing these integrals the following cases should be distinguished:

$$
\begin{aligned}
& \left(G_{s r}\right)_{i l} \\
& \quad= \begin{cases}0 & \text { if } x_{l}<\tau_{1} \\
\int_{\tau_{1}}^{x_{l}} k_{s r}\left(x_{l}, t\right) d t & \text { if } \tau_{1} \leq x_{l}<\tau_{2} \\
\int_{\tau_{1}}^{\tau_{2}} k_{s r}\left(x_{l}, t\right) d t-\int_{\tau_{2}}^{x_{l}} k_{s r}\left(x_{l}, t\right) d t & \text { if } \tau_{2} \leq x_{l}<\tau_{3} \\
\int_{\tau_{1}}^{\tau_{2}} k_{s r}\left(x_{l}, t\right) d t-\int_{\tau_{2}}^{\tau_{3}} k_{s r}\left(x_{l}, t\right) d t & \text { if } x_{l} \geq \tau_{3} .\end{cases}
\end{aligned}
$$

Next we will evaluate the wavelet coefficients $a_{i}$ and $b_{i}$ in the following way.

Collocation Method. Satisfying (27) and (28) only at the collocation points (7) we get a system of linear equations

$$
\begin{array}{r}
\sum_{i=1}^{2 M}\left[a_{i}\left(h_{i}\left(t_{l}\right)-\left(G_{11}\right)_{i}\left(x_{l}\right)\right)-b_{i}\left(G_{12}\right)_{i}\left(x_{l}\right)\right]=f_{1}\left(x_{l}\right), \\
\sum_{i=1}^{2 M}\left[b_{i}\left(h_{i}\left(t_{l}\right)-\left(G_{22}\right)_{i}\left(x_{l}\right)\right)-a_{i}\left(G_{21}\right)_{i}\left(x_{l}\right)\right]=f_{2}\left(x_{l}\right), \\
l=1,2, \ldots, 2 M .
\end{array}
$$

The matrix form of this system is

$$
a\left(H-G_{11}\right)-b G_{12}=F_{1}, \quad b\left(H-G_{22}\right)-a G_{21}=F_{2} \text {. }
$$


Example 4. Consider the following (SLVIEs) [17]:

$$
\begin{aligned}
& u(x)-\int_{0}^{x} x t u(t) d t-\int_{0}^{x}(x+t) v(t) d t \\
& =e^{2 x}\left(-\frac{1}{2} x^{2}+\frac{1}{4} x+1\right)+e^{-2 x}\left(x+\frac{1}{4}\right)-\frac{3}{4} x-\frac{1}{4} \\
& v(x)-\int_{0}^{x}(x-t) u(t) d t-\int_{0}^{x}(x+t)^{2} v(t) d t \\
& =e^{-2 x}\left(2 x^{2}+x+\frac{5}{4}\right)-\frac{1}{4} e^{2 x}-\frac{1}{2} x^{2} .
\end{aligned}
$$

Carrying out the integration in (28) we obtain

$$
\begin{aligned}
& \left(G_{11}\right)_{i l} \\
& \quad= \begin{cases}0 & \text { for } x_{l}<\tau_{1} \\
\frac{1}{2}\left(x_{l}^{3}-x_{l} \tau_{1}^{2}\right) & \text { for } \tau_{1} \leq x_{l}<\tau_{2} \\
-\frac{1}{2} x_{l}\left(x_{l}^{2}+\tau_{1}^{2}-2 \tau_{2}^{2}\right) & \text { for } \tau_{2} \leq x_{l}<\tau_{3} \\
-\frac{1}{2} x_{l}\left(\tau_{1}^{2}-2 \tau_{2}^{2}+\tau_{3}^{2}\right) & \text { for } x_{l} \geq \tau_{3},\end{cases}
\end{aligned}
$$

$\left(G_{12}\right)_{i l}$

$$
\left\{\begin{array}{l}
0 \quad \text { for } x_{l}<\tau_{1} \\
\frac{1}{2}\left(x_{l}-\tau_{1}\right)\left(3 x_{l}+\tau_{1}\right) \\
\quad \text { for } \tau_{1} \leq x_{l}<\tau_{2} \\
-\frac{3 x_{l}^{2}}{2}-\frac{\tau_{1}^{2}}{2}-x_{l}\left(\tau_{1}-2 \tau_{2}\right)+\tau_{2}^{2} \\
\text { for } \tau_{2} \leq x_{l}<\tau_{3} \\
-\frac{\tau_{1}^{2}}{2}+\tau_{2}^{2}-\frac{\tau_{3}^{2}}{2}-x_{l}\left(\tau_{1}-2 \tau_{2}+\tau_{3}\right) \\
\text { for } x_{l} \geq \tau_{3},
\end{array}\right.
$$

$\left(G_{21}\right)_{i l}$

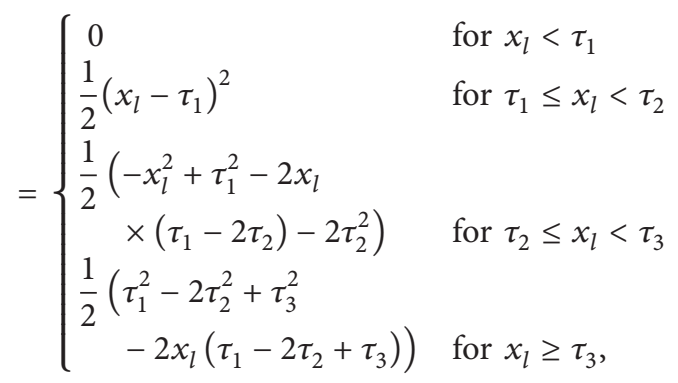

$\left(G_{22}\right)_{i l}$

$$
\left\{\begin{array}{l}
0 \quad \text { for } x_{l}<\tau_{1} \\
\frac{8 x_{l}^{3}}{3}-\frac{1}{3}\left(x_{l}+\tau_{1}\right)^{3} \\
\quad \text { for } \tau_{1} \leq x_{l}<\tau_{2} \\
\frac{1}{3}\left(-8 x_{l}^{3}-\left(x_{l}+\tau_{1}\right)^{3}+2\left(x_{l}+\tau_{2}\right)^{3}\right) \\
\quad \text { for } \tau_{2} \leq x_{l}<\tau_{3} \\
\frac{1}{3}\left(-\left(x_{l}+\tau_{1}\right)^{3}+2\left(x_{l}+\tau_{2}\right)^{3}-\left(x_{l}+\tau_{3}\right)^{3}\right) \\
\quad \text { for } x_{l} \geq \tau_{3} .
\end{array}\right.
$$

We apply collocation method, and vectors $a_{i}$ and $b_{i}$ can be calculated from (30); the functions $u(x)$ and $v(x)$ are evaluated from (25).

Computations were carried out for different values of $J$ (see Table 4 and Figure 4 ). These results were compared with the exact solution $u(x)=e^{2 x}$ and $v(x)=e^{-2 x}$.

The accuracy of the results was estimated by the error function for $u(x)$ and $v(x)$ :

$$
\begin{aligned}
& e_{J}=\max _{1 \leq l \leq 2 M}\left|u\left(x_{l}\right)-u_{e x}\left(x_{l}\right)\right|, \\
& e_{J}=\max _{1 \leq l \leq 2 M}\left|v\left(x_{l}\right)-v_{e x}\left(x_{l}\right)\right|,
\end{aligned}
$$

where $x_{l}$ is defined by (7).

Example 5. Consider the following (SLVIEs) $[4,18]$ :

$u(x)$

$$
\begin{aligned}
= & -1-x \cos (x)^{2}+2 \cos (x)+\sin (x)\left(1-\frac{1}{2} x+\cos (x)\right) \\
& +\int_{0}^{x}((\sin (x-t)-1) u(t)+(1-t \cos (x)) v(t)) d t
\end{aligned}
$$

$v(x)=-x+\sin (x)+\int_{0}^{x}(u(t)+(x-t) v(t)) d t$

Carrying out the integration in (28) we obtain

$\left(G_{11}\right)_{i l}$

$$
= \begin{cases}0 & \text { for } x_{l}<\tau_{1} \\ 1-\cos \left[x_{l}-\tau_{1}\right]-x_{l}+\tau_{1} & \text { for } \tau_{1} \leq x_{l}<\tau_{2} \\ -1-\cos \left[x_{l}-\tau_{1}\right]+2 \cos \left[x_{l}-\tau_{2}\right] & \\ \quad+x_{l}+\tau_{1}-2 \tau_{2} & \text { for } \tau_{2} \leq x_{l}<\tau_{3} \\ -\cos \left[x_{l}-\tau_{1}\right]+2 \cos \left[x_{l}-\tau_{2}\right] & \\ \quad-\cos \left[x_{l}-\tau_{3}\right]+\tau_{1}-2 \tau_{2}+\tau_{3} & \text { for } x_{l} \geq \tau_{3},\end{cases}
$$

$\left(G_{12}\right)_{i l}$

$$
= \begin{cases}0 & \text { for } x_{l}<\tau_{1} \\ x_{l}-\tau_{1}+\frac{1}{2} \cos \left[x_{l}\right]\left(-x_{l}^{2}+\tau_{1}^{2}\right) & \text { for } \tau_{1} \leq x_{l}<\tau_{2} \\ -x_{l}-\tau_{1}+2 \tau_{2} & \\ +\frac{1}{2} \cos \left[x_{l}\right]\left(x_{l}^{2}+\tau_{1}^{2}-2 \tau_{2}^{2}\right) & \text { for } \tau_{2} \leq x_{l}<\tau_{3} \\ \frac{1}{2}\left(-2\left(\tau_{1}-2 \tau_{2}+\tau_{3}\right)\right. & \\ \left.\quad+\cos \left[x_{l}\right]\left(\tau_{1}^{2}-2 \tau_{2}^{2}+\tau_{3}^{2}\right)\right) & \text { for } x_{l} \geq \tau_{3},\end{cases}
$$

$\left(G_{21}\right)_{i l}$

$$
= \begin{cases}0 & \text { for } x_{l}<\tau_{1} \\ x_{l}-\tau_{1} & \text { for } \tau_{1} \leq x_{l}<\tau_{2} \\ -x_{l}-\tau_{1}+2 \tau_{2} & \text { for } \tau_{2} \leq x_{l}<\tau_{3} \\ -\tau_{1}+2 \tau_{2}-\tau_{3} & \text { for } x_{l} \geq \tau_{3},\end{cases}
$$




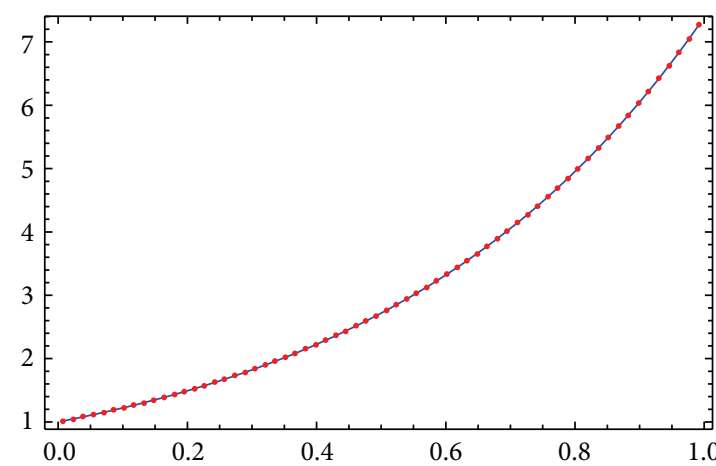

Exact solution

- Numerical solution

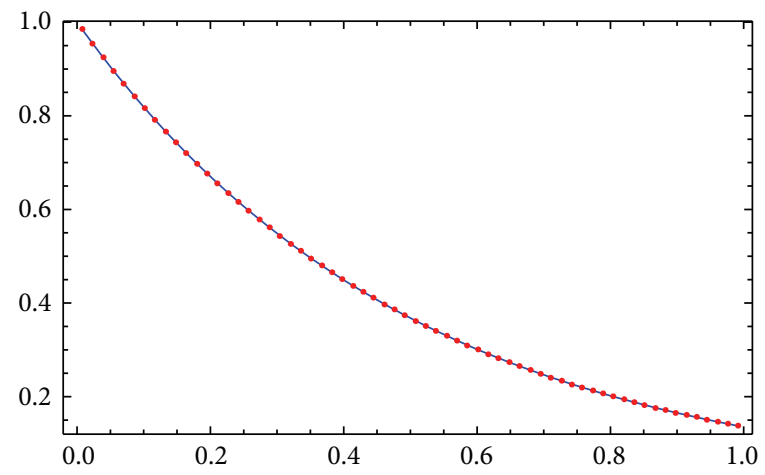

- Exact solution

- Numerical solution

(a)

(b)

Figure 4: (a) Exact and Haar wavelet solution of $u(x)$ at $J=5$. (b) Exact and Haar wavelet solution of $v(x)$ at $J=5$.

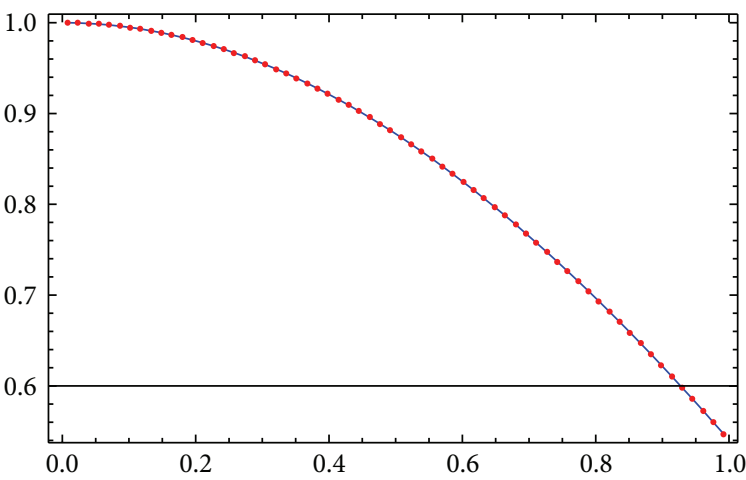

— Exact solution

- Numerical solution

(a)

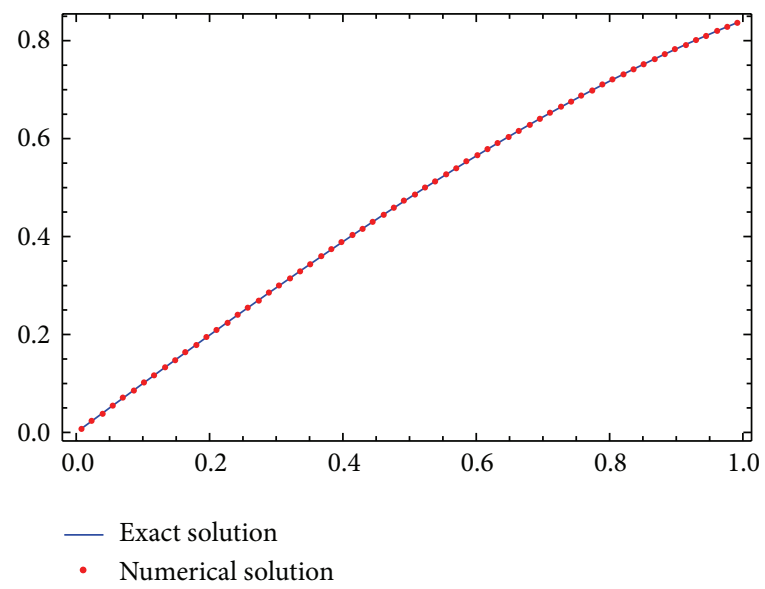

(b)

Figure 5: (a) Exact and Haar wavelet solution of $u(x)$ at $J=5$. (b) Exact and Haar wavelet solution of $v(x)$ at $J=5$.

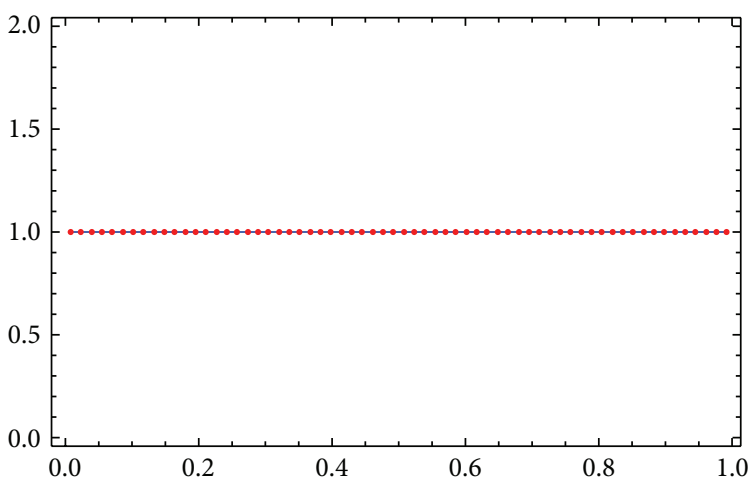

- Exact solution

- Numerical solution

(a)

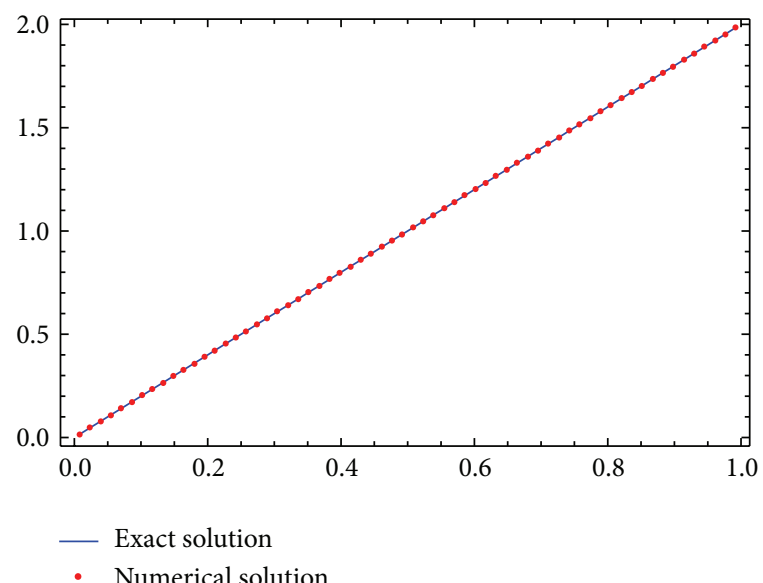

(b)

Figure 6: (a) Exact and Haar wavelet solution of $u(x)$ at $J=5$. (b) Exact and Haar wavelet solution of $v(x)$ at $J=5$. 


$$
\begin{aligned}
& \left(G_{22}\right)_{i l} \\
& =\left\{\begin{array}{l}
0 \quad \text { for } x_{l}<\tau_{1} \\
\frac{1}{2}\left(x_{l}-\tau_{1}\right)^{2} \\
\text { for } \tau_{1} \leq x_{l}<\tau_{2} \\
\frac{1}{2}\left(-x_{l}^{2}+\tau_{1}^{2}-2 x_{l}\left(\tau_{1}-2 \tau_{2}\right)-2 \tau_{2}^{2}\right) \\
\text { for } \tau_{2} \leq x_{l}<\tau_{3} \\
\frac{1}{2}\left(\tau_{1}^{2}-2 \tau_{2}^{2}+\tau_{3}^{2}-2 x_{l}\left(\tau_{1}-2 \tau_{2}+\tau_{3}\right)\right) \\
\text { for } x_{l} \geq \tau_{3} .
\end{array}\right.
\end{aligned}
$$

We apply collocation method, and vectors $a_{i}$ and $b_{i}$ can be calculated from (29); the functions $u(x)$ and $v(x)$ are evaluated from (25).

Computations were carried out for different values of $J$ (see Table 5 and Figure 5 ). These results were compared with the exact solution $u(x)=\cos (x)$ and $v(x)=\sin (x)$.

Example 6. Consider the following (SLVIEs):

$$
u(x)=1-x^{2}+\int_{0}^{x} v(t) d t, \quad v(x)=x+\int_{0}^{x} u(t) d t
$$

Carrying out the integration in (28) we obtain

$$
\begin{aligned}
& \left(G_{12}\right)_{i l}= \begin{cases}0 & \text { for } x_{l}<\tau_{1} \\
x_{l}-\tau_{1} & \text { for } \tau_{1} \leq x_{l}<\tau_{2} \\
-x_{l}-\tau_{1}+2 \tau_{2} & \text { for } \tau_{2} \leq x_{l}<\tau_{3} \\
-\tau_{1}+2 \tau_{2}-\tau_{3} & \text { for } x_{l} \geq \tau_{3},\end{cases} \\
& \left(G_{21}\right)_{i l}= \begin{cases}0 & \text { for } x_{l}<\tau_{1} \\
x_{l}-\tau_{1} & \text { for } \tau_{1} \leq x_{l}<\tau_{2} \\
-x_{l}-\tau_{1}+2 \tau_{2} & \text { for } \tau_{2} \leq x_{l}<\tau_{3} \\
-\tau_{1}+2 \tau_{2}-\tau_{3} & \text { for } x_{l} \geq \tau_{3} .\end{cases}
\end{aligned}
$$

We apply collocation method, and vectors $a_{i}$ and $b_{i}$ can be calculated from (29); the functions $u(x)$ and $v(x)$ are evaluated from (25).

Computations were carried out for different values of $J$ (see Table 6 and Figure 6). These results were compared with the exact solution $u(x)=1$ and $v(x)=2 x$.

\section{Conclusion}

In this work the Haar wavelet method for solution of linear systems integral equations is proposed. A method of solution which is applicable for different kind of integral equations, Fredholm and Volterra systems of integral equations, is worked out. The solution is based on the collocation techniques which are proposed. The elaborated method is very simple and, as it follows from the test problems, high precision of results can be obtained with a small number of calculation points. The calculations show that by doubling the number of the calculation points the error function decreases. This result follows also from analytical considerations. It
TABLE 4: Error of $u(x)$ and $v(x)$ of Example 4.

\begin{tabular}{cccc}
\hline$J$ & $2 M$ & Error of function $u(x)$ & Error of function $v(x)$ \\
\hline 2 & 8 & $1.51 E-2$ & $9.43 E-3$ \\
3 & 16 & & \\
4 & 32 & & \\
5 & 64 & $3.14 E-4$ & $1.91 E-4$ \\
\hline
\end{tabular}

TABLE 5: Error of $u(x)$ and $v(x)$ of Example 5.

\begin{tabular}{cccc}
\hline$J$ & $2 M$ & Error of function $u(x)$ & Error of function $v(x)$ \\
\hline 2 & 8 & $1.74 E-3$ & $1.94 E-3$ \\
3 & 16 & $4.78 E-4$ & $5.07 E-4$ \\
4 & 32 & $1.20 E-4$ & $1.29 E-4$ \\
5 & 64 & $30.3 E-5$ & $3.27 E-5$ \\
\hline
\end{tabular}

TABLE 6: Error of $u(x)$ and $v(x)$ of Example 6.

\begin{tabular}{cccc}
\hline$J$ & $2 M$ & Error of function $u(x)$ & Error of function $v(x)$ \\
\hline 2 & 8 & $5.76 E-3$ & $4.23 E-3$ \\
3 & 16 & $1.47 E-3$ & $1.10 E-3$ \\
4 & 32 & $2.72 E-4$ & $2.82 E-4$ \\
5 & 64 & $9.36 E-5$ & $7.09 E-5$ \\
\hline
\end{tabular}

should be noted that in the case of Haar wavelets we have to solve systems of linear equations with a smaller condition number as for other methods based on piecewise constant approximation.

\section{Conflict of Interests}

The authors declare that there is no conflict of interests regarding the publication of this paper.

\section{References}

[1] S. S. Ray and P. K. Sahu, "Numerical methods for solving Fredholm integral equations of second kind," Abstract and Applied Analysis, vol. 2013, Article ID 426916, 17 pages, 2013.

[2] H. Sadeghi Goghary, S. H. Javadi, and E. Babolian, "Restarted Adomian method for system of nonlinear Volterra integral equations," Applied Mathematics and Computation, vol. 161, no. 3, pp. 745-751, 2005.

[3] K. Maleknejad, N. Aghazadeh, and M. Rabbani, "Numerical solution of second kind Fredholm integral equations system by using a Taylor-series expansion method," Applied Mathematics and Computation, vol. 175, no. 2, pp. 1229-1234, 2006.

[4] M. Rabbani, K. Maleknejad, and N. Aghazadeh, "Numerical computational solution of the Volterra integral equations system of the second kind by using an expansion method," Applied Mathematics and Computation, vol. 187, no. 2, pp. 1143-1146, 2007.

[5] K. Maleknejad and F. Mirzaee, "Numerical solution of linear Fredholm integral equations system by rationalized Haar functions method," International Journal of Computer Mathematics, vol. 80, no. 11, pp. 1397-1405, 2003.

[6] M. Javidi and A. Golbabai, "A numerical solution for solving system of Fredholm integral equations by using homotopy 
perturbation method," Applied Mathematics and Computation, vol. 189, no. 2, pp. 1921-1928, 2007.

[7] M. C. de Bonis and C. Laurita, "Numerical treatment of second kind Fredholm integral equations systems on bounded intervals," Journal of Computational and Applied Mathematics, vol. 217, no. 1, pp. 64-87, 2008.

[8] G. Hariharan and K. Kannan, "An overview of Haar wavelet method for solving differential and integral equations," World Applied Sciences Journal, vol. 23, no. 12, pp. 1-14, 2013.

[9] G. Hariharan and K. Kannan, "Review of wavelet methods for the solution of reaction-diffusion problems in science and engineering," Applied Mathematical Modelling, vol. 38, no. 3, pp. 799-813, 2014.

[10] J. Rashidinia and M. Zarebnia, "Convergence of approximate solution of system of Fredholm integral equations," Journal of Mathematical Analysis and Applications, vol. 333, no. 2, pp. 12161227, 2007.

[11] K. Ivaz and B. S. Mostahkam, "Newton-Tau numerical solution of a system of nonlinear Fredholm integral equations of second kind," Applied and Computational Mathematics, vol. 5, no. 2, pp. 201-208, 2006.

[12] E. Babolian and M. Mordad, "A numerical method for solving systems of linear and nonlinear integral equations of the second kind by hat basis functions," Computers and Mathematics with Applications, vol. 62, no. 1, pp. 187-198, 2011.

[13] K. Maleknejad, M. Shahrezaee, and H. Khatami, "Numerical solution of integral equations system of the second kind by block-pulse functions," Applied Mathematics and Computation, vol. 166, no. 1, pp. 15-24, 2005.

[14] K. Maleknejad and A. Salimi Shamloo, "Numerical solution of singular Volterra integral equations system of convolution type by using operational matrices," Applied Mathematics and Computation, vol. 195, no. 2, pp. 500-505, 2008.

[15] H. A. Zedan and E. El Adrous, "The application of the homotopy perturbation method and the homotopy analysis method to the generalized Zakharov equations," Abstract and Applied Analysis, vol. 2012, Article ID 561252, 19 pages, 2012.

[16] H. H. Sorkun and S. Yalçinbaş, "Approximate solutions of linear Volterra integral equation systems with variable coefficients," Applied Mathematical Modelling, vol. 34, no. 11, pp. 3451-3464, 2010.

[17] J. Biazar and M. Eslami, "Modified HPM for solving systems of Volterra integral equations of the second kind," Journal of King Saud University (Science), vol. 23, no. 1, pp. 35-39, 2011.

[18] M. I. Berenguer, D. Gámez, A. I. Garralda-Guillem, M. Ruiz Galán, and M. C. Serrano Pérez, "Biorthogonal systems for solving Volterra integral equation systems of the second kind," Journal of Computational and Applied Mathematics, vol. 235, no. 7, pp. 1875-1883, 2011. 


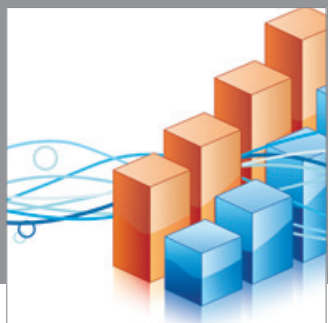

Advances in

Operations Research

mansans

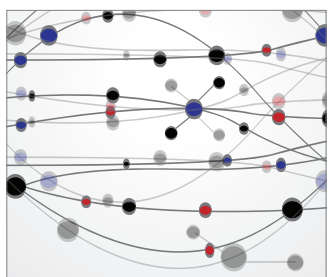

The Scientific World Journal
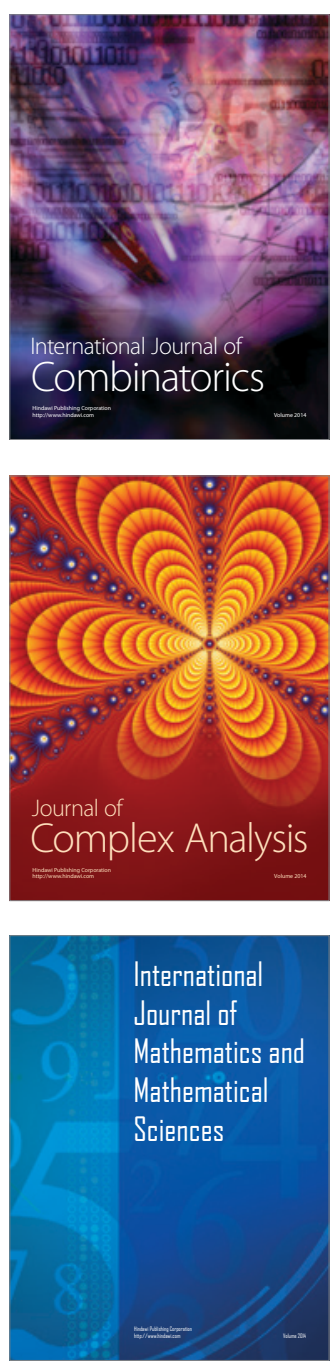
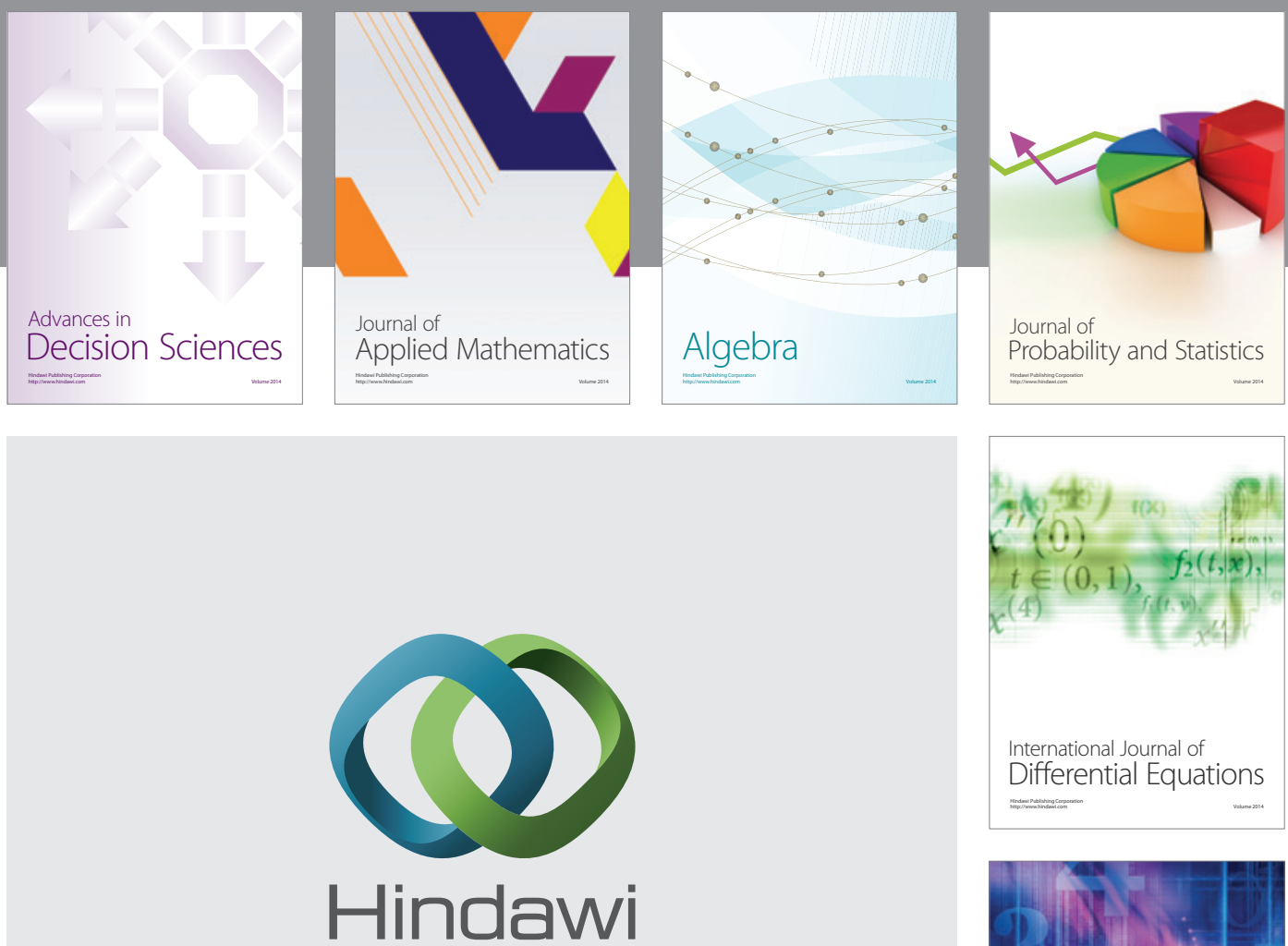

Submit your manuscripts at http://www.hindawi.com
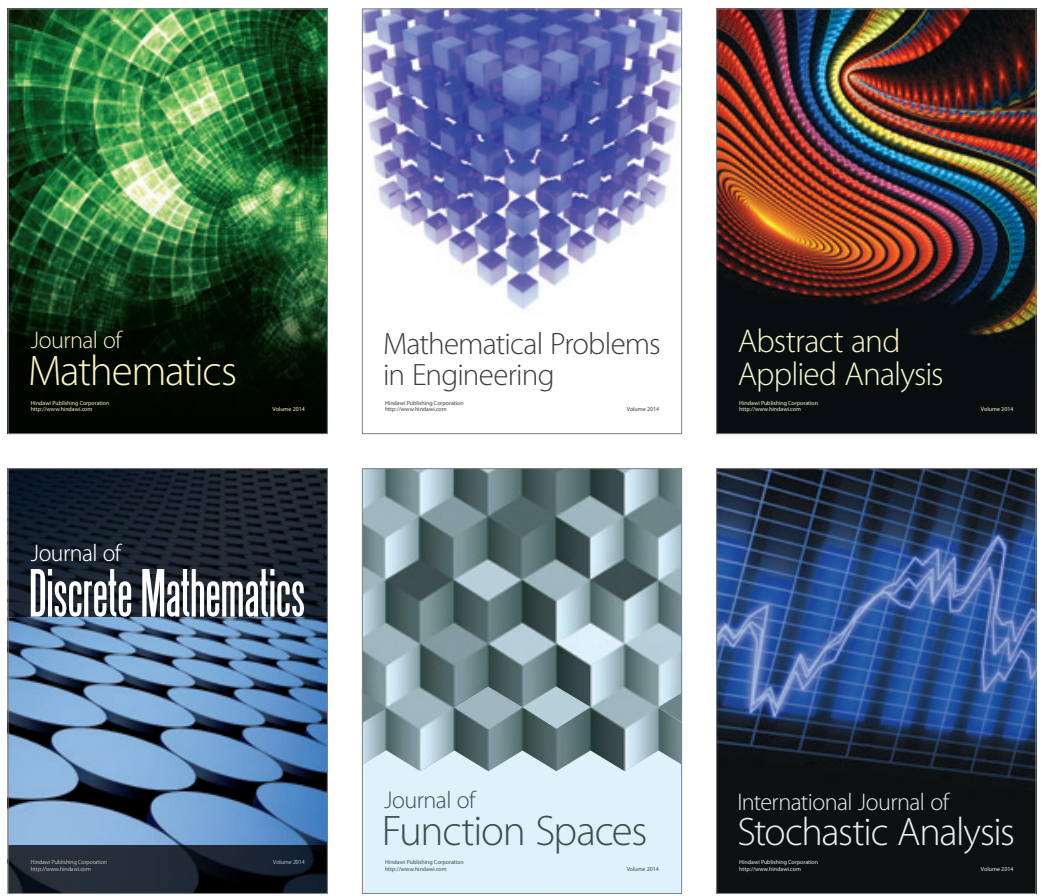

Journal of

Function Spaces

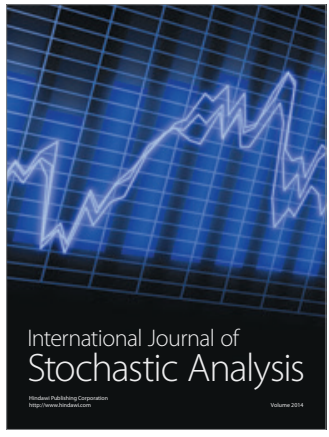

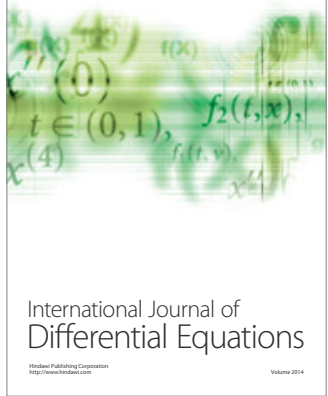
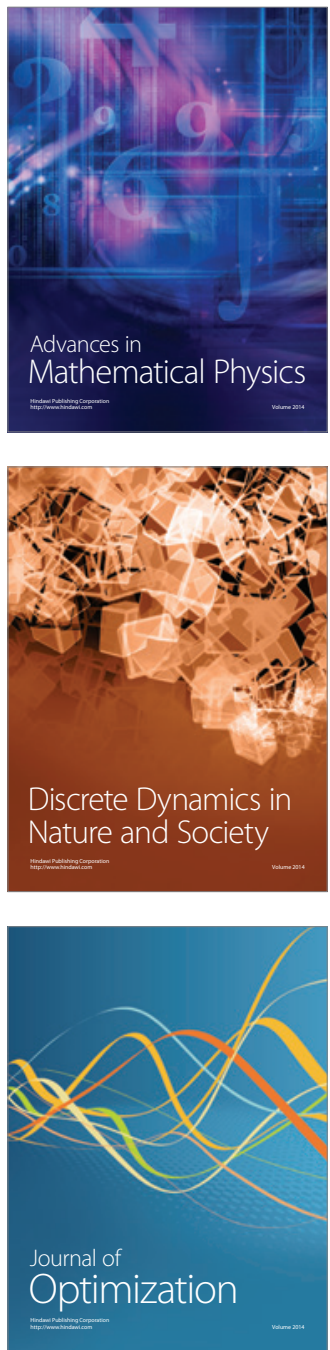